\title{
Capacidad de regeneración natural del bosque de manglar del Estero Tortuga, Osa, Puntarenas, Costa Rica
}

\author{
Elena Vargas-Fonseca \\ Fundación para la Investigación y Conservación de Playa Tortuga, Puntarenas, Costa Rica; elena.vargasf@gmail.com
}

\author{
Recibido 11-VIII-2014. Corregido 20-XI-2014. Aceptado 22-XII-2014.
}

\begin{abstract}
Natural regeneration capacity of the mangrove forest of Tortuga Estuary, Osa, Puntarenas, Costa Rica. The study was conducted from February 2011 to January 2012, in the mangrove forest of Tortuga Estuary. A total of six parcels, each measuring $9 \mathrm{~m}^{2}$, were established in the area, to evaluate the survival and growth rates of the mangrove seedlings. By the end of the study all parcels presented $100 \%$ mortality, indicating that the area is not recruiting young trees and therefore is not regenerating naturally. The results of this study suggest that the low salinity of the water table and the high rate of deposition of sediments, are the causes of the low survival rate of the seedlings and could also be affecting the adult individuals. The adult trees presented deteriorated health signs such as decomposition of their stilt roots and total loss of foliage. The borders of this ecosystem were georeferenced and compared with the official map that declares the Natural Patrimony of the Country, to determine areas that should be protected under this category. In general the mangrove forest of this estuary can be divided in two zones: the first one is characterized by a mature forest of mainly two species: Rhizophora racemosa (G. Mey.) and Pelliciera rhizophorae (Triana \& Planch.). The second one is located in the banks of sand of the estuary, where the dominant specie is Laguncularia racemosa (C.F. Gaertn) in young stages. Both of these areas include mangrove ecosystems that are not included in the Natural Patrimony category. Rev. Biol. Trop. 63 (Suppl. 1): 209-218. Epub 2015 April 01.
\end{abstract}

Key words: Survival, seedling, Rhizophora racemosa, Pelliciera rhizophorae, restoration, Laguncularia racemosa, Patrimonio Natural del Estado.

Los manglares se distribuyen alrededor del mundo y se encuentran principalmente en los trópicos y subtrópicos (Duke, 1992; Spalding, Kainuma \& Collins, 2010). El 70\% de los manglares de América se encuentra en la costa atlántica (Yáñez-Arancibia \& Lara-Domínguez, 1999) mientras que el $99 \%$ de los manglares de Costa Rica se ubican a lo largo de la costa pacífica (Pizarro \& Angulo, 1994), donde los manglares del Humedal Nacional Térraba-Sierpe (HNTS) cubren una extensión de 14637 ha, lo cual representa alrededor del $40 \%$ de todos los bosques de manglar en nuestro país (CórdobaMuñoz, Romero-Araya \& Windevoxhel-Lora, 1998; Lahmann-Zeledón, 1999).

Los bosque de mangle son uno de los ecosistemas más biodiversos del mundo (Spalding et al., 2010). Estos desempeñan funciones importantes como protección ante la erosión, retención de sedimentos, contribución de material orgánico para la productividad primaria y secundaria en las aguas costeras, proporcionan alimento y refugio para muchos organismos acuáticos a lo largo de sus diferentes etapas de la vida, y protege los recursos genéticos de diferentes especies (Pizarro \& Angulo, 1994; Pizarro, Piedra, Bravo, Asch \& Asch, 2004).

Estos ecosistemas han sido alterados debido a factores antropogénicos tales como extracción de madera, contaminación, relleno de tierras, drenaje de los cuerpos de agua, entre otros (Frazier, 1999; Villela, 1999; UICN, 2002; Ellison, 2004). Durante las dos últimas décadas se ha producido una reducción del $35 \%$ en las zonas de manglares en todo el mundo (Valiela, Bowen \& York, 2001), por 
lo tanto es de vital importancia conservar los ecosistemas remanentes y facilitar su restauración. No obstante, en ecosistemas degradados, la regeneración natural de los manglares es un proceso que se puede ver severamente impedido (Said-Mohamed, Neukermans, GitunduKairo, Dahdouh-Guebas \& Koedam, 2009; Bosire et al., 2013) especialmente si las condiciones ambientales son modificadas (Upadhyay, Ranjan \& Singh, 2002).

Actualmente el Humedal Nacional Térraba Sierpe cubre una superficie de aproximadamente 14 700ha, sin embargo el ecosistema de humedales que se ubica en la zona presenta una extensión de aproximadamente $39000 \mathrm{ha}$ (Uribe, 2010). Por lo tanto gran parte de estos humedales no gozan el mismo grado de protección. Además, una de las amenazas más importantes señaladas por diversos autores es la sedimentación (Umaña-Villalobos \& Springer, 2006; Flores-Guillen, 2001 citado en Sierra et al., 2007; Castillo \& Arguedas, 2007; Rojas \& Rodríguez, 2008), la cual afecta la totalidad del ecosistema, sin discriminar los grados de protección presentes o ausentes.

Los manglares del Estero Tortuga, son parte de estas áreas que no se encuentran dentro de los límites oficiales del HNTS, y presentan fuentes de degradación como alta tasa de deposición de sedimentos, ocupación ilegal, incendios y cacería (obs. pers.). Además los límites de este ecosistema no se encuentran debidamente demarcados, lo que ha facilitado la ocurrencia de eventos de deforestación no registrados.

Según la legislación de Costa Rica, los manglares forman parte del Patrimonio Natural del Estado (MINAET, 2010), ya que son humedales importantes para la conservación de los recursos naturales. El Sistema Nacional de Áreas de Conservación (SINAC) ha realizado esfuerzos por registrar estos ecosistemas con el fin de actualizar el Inventario de Humedales de Costa Rica. A través de este estudio, La Reserva Playa Tortuga pretende colaborar con estos esfuerzos, por medio de la determinación de la capacidad de regeneración natural del bosque de manglar del Estero Tortuga. Además se realizó un análisis comparativo de las zonas de manglar declaradas Patrimonio Natural de Estado y los bosques de manglar del Estero Tortuga, con el fin de proveer información actualizada sobre la extensión de este ecosistema y así colaborar con los esfuerzos de conservación realizados por el SINAC.

\section{MATERIALES Y MÉTODOS}

Descripción del área de estudio: El Estero Tortuga se encuentra en las coordenadas $9^{\circ} 07^{\prime}$ $\mathrm{N}-83^{\circ} 66^{\prime} \mathrm{W}$, en el distrito de Puerto Cortés, Osa, Puntarenas, Costa Rica. La desembocadura del río consiste en un sistema de canales que se ve influido por los movimientos de la marea. El área incluye diferentes tipos de hábitat: bosques de mangle, bosques riparios, bosques secundarios y áreas deforestadas dominadas por pastos y parches de plantaciones agrícolas de uso doméstico. El bosque de mangle está dominado por las especies Rhizophora racemosa (G. Mey.), Pelliciera rhizophorae (Triana \& Planch.) y el helecho Acrostichum aureum (L.) aunque también se encuentran zonas en regeneración de Laguncularia racemosa (C. F.Gaertn) en secciones adyacentes al canal principal del Estero Tortuga (obs. pers.).

El área se encuentra entre cinco y diez metros sobre el nivel del mar, con temperaturas entre $21.1^{\circ} \mathrm{C}$ y $33.6^{\circ} \mathrm{C}$ y una precipitación anual de $4953.9 \mathrm{~mm}$, con una estación seca definida a partir de diciembre a abril (IMN, 2012).

Metodología: El área de estudio se definió en base a la delimitación de Patrimonio Natural del Estado realizada en el 2009 por el Área de Conservación de Osa, quienes utilizan la siguiente nomenclatura B: bosque, $\mathrm{H}$ : humedal, NP: no patrimonio, seguido por un número, con el fin de clasificar diferentes áreas de acuerdo al tipo de hábitat (ACOSA, 2009).

Se recorrieron los márgenes del bosque de mangle del estero Tortuga, ubicados entre el Río Balso y el extremo norte de Playa Tortuga. Se utilizaron los criterios definidos por la legislación nacional para la clasificación de humedales: presencia de vegetación hidrófila, suelos 
hídricos y condición hídrica (MINAET, 2010) para definir a nivel cualitativo el límite de los humedales ubicados en los sectores NP1, NP2, NP3, H1, H2 y B2. Para ello se utilizó un GPS Garmin y se produjo un mapa comparativo entre los límites actuales del bosque de manglar y el mapa oficial mencionado.

Entre los meses de febrero 2011 y enero 2012, se establecieron un total de seis parcelas fijas, con dimensiones de nueve metros cuadrados cada una, ubicadas al azar en zonas con presencia de plántulas de mangle de las dos especies presentes en el ecosistema. En cada parcela, se marcó cada uno de los individuos con altura menor a $0.5 \mathrm{~m}$. Se registró su especie y durante el primer semestre del estudio, se midió mensualmente la altura de cada plántula desde la base del hipocótilo a la yema terminal. Las plántulas que se encontraban muertas se reconocieron por su estado de descomposición y se registraron como tales. Además se realizó una descripción cualitativa de cada parcela, incluyendo la presencia de otras especies de plantas, el tipo de suelo (arcilloso, arenoso, lodoso) y grado de exposición al sol (soleado, parcialmente soleado, bajo sombra). Con los datos recolectados, se calculó la tasa de supervivencia $\left(\mathrm{TS}=100-\mathrm{N}_{\text {muertos }} * 100 / \mathrm{N}_{\text {inicial }}\right)$ y la tasa de crecimiento mensual de cada una de las parcelas $\left(T C M=\Sigma_{:} h_{n, \text { mes } x}-h_{n, \text { mes x-1 }} / N\right)$. Se realizó una última revisión de las parcelas en enero del 2012 y se registraron las mismas variables.

Durante el primer semestre del estudio y mensualmente se midieron tres variables físicas: tasa de deposición de sedimentos, nivel freático y su salinidad, lo cual se realizó utilizando un tubo de PVC de un metro de altura y $15 \mathrm{~cm}$ de diámetro, con agujeros de $0.5 \mathrm{~cm}$ de diámetro a lo largo que permiten el movimiento del agua de acuerdo al nivel freático. Este fue enterrado verticalmente $0.85 \mathrm{~m}$ bajo tierra y cubierto en la parte superior para evitar la acumulación de lluvia dentro del tubo. La sección restante de $15 \mathrm{~cm}$ de altura que quedó expuesta sobre el nivel del suelo, se utilizó como objeto control para medir la tasa mensual de deposición de sedimentos (TD). Para medir el nivel freático, se utilizó una estaca calibrada, la cual se insertó dentro del tubo. Se tomaron muestras del agua acumulada dentro del tubo y se utilizó un refractómetro de mano para medir la salinidad.

Con el fin de establecer la tasa de crecimiento promedio (TCP) de cada parcela en un ecosistema con altas tasas de deposición de sedimentos, se promediaron todos los TCM de cada parcela y se sumó la tasa de deposición de sedimentos. La TCP de la parcela cuatro se determinó utilizando los datos de febrero a junio, ya que posterior a este mes, todas las plántulas habían perecido.

\section{RESULTADOS}

El mapa generado por medio de sistemas de información geográfica (Fig. 1) muestra en polígonos sólidos los límites actuales del bosque de manglar del Estero Tortuga, registrados durante este estudio, y en líneas continuas la categorización del Patrimonio Natural del Estado realizada por ACOSA (2009) B: bosque, H: humedales y NP: no patrimonio.

En términos de área, se estimó que la delimitación actual de Patrimonio Natural del Estado no reconoce la presencia de al menos 3.03ha de bosque de manglar en el Estero Tortuga, lo cual corresponde a un $6.8 \%$ del total, ubicados en los polígonos NP1, NP2 y NP3. Además aproximadamente $8.05 \mathrm{ha}$, correspondientes a $18.0 \%$ del total, ubicadas en los polígonos NP3 y NP10 corresponden a bosque ripario en diferentes estados de regeneración que no fueron evaluados en este estudio. Se confirmó que el 100\% del área ubicada en los polígonos B2, H1 y H2 corresponden a zonas cubiertas por bosque, tanto de manglar como continental. Finalmente el borde oeste del canal principal, colindante con Playa Tortuga, se encontró colonizado en su totalidad por individuos de $L$. racemosa de portes menores a dos metros de altura, cuya extensión no fue estimada (Fig. 1).

El bosque de manglar del Estero Tortuga se puede dividir en dos zonas: la primera se encuentra en las zonas H2 y B2 (Fig. 1), donde se establecieron las parcelas de plántulas de 




Fig. 1. Mapa comparativo entre la delimitación del Patrimonio Natural del Estado (B: bosque, H: humedales y NP: no patrimonio) y los límites actuales del bosque de manglar del Estero Tortuga.

Fig. 1. Comparative map between the delimitation of the National Patrimony (B: forest, H: wetland y NP: not patrimony) and the current border of the mangrove forest of the Tortuga Estuary.

este estudio. Esta zona se caracteriza por un bosque de manglar conformado principalmente por las especies $R$. racemosa y $P$. rhizophorae y una cobertura del sotobosque por parte de $A$. aureum que alcanza el $100 \%$ en algunos sectores del ecosistema. La presencia de al menos 10 individuos adultos de $R$. racemosa, con una altura mayor a $15 \mathrm{~m}$, ubicados en un trayecto de $200 \mathrm{~m}$, con raíces en proceso de descomposición y/o ausencia total de follaje, resalta en el paisaje. La segunda zona está conformada por el sector NP3 (Fig. 1) y los bancos de arena colonizados por $L$. racemosa ubicados en colindancia con Playa Tortuga, la cual no está designada como Patrimonio Natural del Estado a pesar de contar con las condiciones escenciales de un humedal (Bravo \& Windevoxhel, 1997).

El nivel freático está influenciado por los cambios de marea, en marea baja se encuentra por debajo de los $85 \mathrm{~cm}$, y por lo tanto no fue determinado, mientras que en marea alta es de $48.2 \mathrm{~cm}$ en promedio ( $\mathrm{DE}=9.26, \mathrm{n}=6$ ). Las muestras de agua tomadas del manto freático presentan una salinidad promedio de $1.5 \mathrm{~g} / \mathrm{L}$ $(\mathrm{DE}=0.5, \mathrm{n}=6)$. La tasa de deposición de sedimentos se estimó en $1.67 \mathrm{~cm} / \mathrm{mes}$ ( $\mathrm{DE}=0.7$ $\mathrm{n}=6$ ), la cual es visible como una capa de arcilla depositada posterior a eventos de inundación causados por mareas extremas o fuertes lluvias.

Las seis parcelas evaluadas fueron monoespecíficas en cuanto a las especies de manglar presentes dentro de cada una: las parcelas 1, 2, 4, 5, 6 siendo de $R$. racemosa y la parcela 3 de $P$. rhizophorae. Estas presentaron un total de plántulas por parcela entre 21 y 89 individuos, para un total de 273 plántulas evaluadas. Durante el primer semestre del estudio, la parcela 1 presentó la tasa de supervivencia más alta, seguida por la parcela 6 , mientras que las parcelas 3 y 5 presentaron la tasa de 
supervivencia más baja, pero las mismas presentaron tasas de crecimiento opuestas: la más alta para la parcela 3 y nula para la parcela 5 . La parcela 2, presentó la segunda tasa de crecimiento más alta pero una tasa de supervivencia relativamente baja. La parcela 4 presentó la tasa de crecimiento más baja y tras un periodo de cuatro meses, una tasa de supervivencia nula (Cuadro 1).

La última visita a las parcelas se realizó transcurrido un año de establecidas, cuando no se encontraron plántulas vivas en ninguna de las parcelas.

\section{DISCUSIÓN}

Patrimonio Natural del Estado en Estero Tortuga: Las diferencias encontradas en la delimitación del Patrimonio Natural del Estado con respecto a este estudio, son importantes de resaltar, ya que su desprotección amenaza otros sectores del ecosistema. Los manglares de la zona NP3 y bancos de arena expuestos en la bajamar dominados por L. racemosa, separan a las zonas H2 Y B2 del canal principal del Estero Tortuga y circundan la desembocadura del Río Tortuga, por lo que la desprotección de esta zona significa una amenaza directa para los humedales aledaños. La zona NP10 y NP3 se encuentra protegida mediante una Reserva Forestal propiedad de Reserva Playa Tortuga, no obstante la venta ilegal de terrenos en Playa Tortuga (Anónimo, com. pers.), y el precarismo es una problemática generalizada en la región costera del Humedal Nacional Térraba Sierpe, que dificulta la adecuada protección de estas zonas.

Las estimaciones realizadas en este estudio sobre el porcentaje de área que no se encuentra clasificado como Patrimonio Natural del Estado son conservadoras. Las secciones adicionales señaladas en el mapa comparativo, corresponden a las áreas que presentan cobertura del dosel por especies de manglar, que fácilmente se distinguen a nivel cualitativo, no obstante un análisis cuantitativo que considere el tipo de suelo, probablemente señalaría que los humedales del Estero Tortuga son aún más extensos. Por ejemplo se observó que las áreas deforestadas adyacentes al bosque de mangle, presentan signos de regeneración e.g. propágulos en proceso de establecimiento, y plántulas en crecimiento; así como signos de reciente deforestación (obs. pers.).

Capacidad de regeneración: En cuanto a las variables físicas estimadas, el nivel del manto freático es relativamente profundo, en

CUADRO 1

Porcentaje de supervivencia y tasa de crecimiento mensual promedio por parcela, durante el periodo febrero-agosto, 2010

TABLE 1

Percentage of survival and average monthly growing rate per parcel, during the period February-August, 2010

\begin{tabular}{|c|c|c|c|c|c|}
\hline Parcela & Condición de la parcela & Especie & $\mathrm{N}_{0}$ & $\begin{array}{c}\text { Porcentaje de } \\
\text { supervivencia (\%) }\end{array}$ & $\begin{array}{l}\text { Tasa de crecimiento } \\
(\mathrm{cm} / \mathrm{mes})\end{array}$ \\
\hline 1 & $\begin{array}{l}\text { Parcialmente soleada, suelo arcilloso. } \\
\text { Ausencia de otras especies }\end{array}$ & Rhizophora racemosa & 32 & 50.00 & 0.46 \\
\hline 2 & $\begin{array}{l}\text { Bajo sombra de, suelo arcilloso. Alta } \\
\text { presencia de } A \text {. aureum }\end{array}$ & Rhizophora racemosa & 54 & 15.38 & 1.50 \\
\hline 3 & $\begin{array}{l}\text { Bajo sombra, suelo arcilloso. } \\
\text { Presencia baja de Musa sp. }\end{array}$ & Pelliciera rhizophorae & 38 & 7.87 & 1.67 \\
\hline 4 & $\begin{array}{l}\text { Parcialmente soleada, suelo arenoso. } \\
\text { Presencia baja de Piper sp. }\end{array}$ & Rhizophora racemosa & 21 & 0 & 0.10 \\
\hline 5 & $\begin{array}{l}\text { Bajo sombra de } R \text {. racemosa, suelo } \\
\text { arenoso. Ausencia de otras especies }\end{array}$ & Rhizophora racemosa & 89 & 7.89 & 0 \\
\hline 6 & $\begin{array}{l}\text { Bajo sombra de } R \text {. racemosa, suelo } \\
\text { arenoso. Ausencia de otras especies }\end{array}$ & Rhizophora racemosa & 39 & 19.35 & 0.75 \\
\hline
\end{tabular}


comparación con otros ecosistemas de manglar, donde en periodos de marea alta es común que la vegetación se encuentre inundada (Pizarro et al., 2004), situación que solamente se presentó en episodios de fuertes lluvias que causaron el desbordamiento del Río Tortuga (obs. pers.). La salinidad del manto freático fue menor a lo esperado para un ecosistema de manglar (McLeod \& Salm, 2006), y su nivel se encuentra en el límite inferior de las aguas ligeramente salinas (USGS, 2014) Esto afecta directamente a las especies que son halófitas como lo son $R$. racemosa y $P$. rhizophorae (Jiménez \& Soto, 1985). Los bajos valores de la salinidad, indican que el manto freático del ecosistema estudiado proviene principalmente del agua fluvial, en combinación con las aguas subterráneas provenientes de los dos ríos aledaños: Tortuga y Balso. Además se encontró que la tasa de deposición de sedimentos en el área de estudio es sumamente alta, en comparación con otros estudios (McLeod \& Salm, 2006; McKee, Cahoon \& Feller, 2007), lo cual es consecuencia de los procesos de erosión que sufre la Fila Costeña y la cuenca del Térraba (ACOSA, Nature Conservacy, UCI \& ELAP, 2008). Considerando que el periodo de estudio se ubica mayormente, durante la época seca del Pacífico sur de Costa Rica, los efectos de dilución del manto freático y acumulación de sedimentos tras el desbordamiento del Río Tortuga encontrados en este estudio, se verán intensificados durante la época lluviosa.

La ausencia de $A$. aureum en la parcela 1 y 6 , permitió que éstas presentaran la tasa de supervivencia más alta, durante los primeros seis meses de seguimiento, ya que este es un helecho invasivo reconocido por su capacidad de limitar la capacidad de regeneración de los ecosistemas de manglar (Srivastava, Keong \& Muktar, 1987; Mainardi-Grellet, 1995). En contraste, la parcela 2 mostraba una alta presencia de $A$. aureum, lo cual disminuye la cantidad de luz que reciben las plántulas y enclaustra a los individuos que se encuentran directamente bajo las hojas del helecho, lo cual explica la baja tasa de supervivencia. La parcela 3 es la única compuesta por plántulas de $P$. rhizophorae, por lo que es posible que las condiciones de alta tasa de deposición de sedimentos y salinidad baja, afecte en mayor medida a esta especie en comparación con las parcelas de $R$. racemosa, causando la menor tasa de supervivencia. En cuanto a la tasa de crecimiento, esta parcela presentó el valor más alto, lo cual podría deberse al tipo de reproducción de esta especie y el tamaño de los cotiledones de esta plántula, los cuales son grandes y contienen alta cantidad de energía que puede ser utilizada por la plántula durante sus primeros estadíos de crecimiento (Duke, 1992). No obstante, los resultados indican que esta cualidad no se traduce a mayores probabilidades de supervivencia, cuando las condiciones no son óptimas para la regeneración natural. Rabinowitz (1978) reporta que en condiciones controladas, cuando los cotiledones de $P$. rhizophorae se encuentran sumergidos bajo el lodo, estos presentan una mortalidad del 100\%, lo cual es coincidente con los hallazgos de este estudio.

La parcela 4 fue monitoreada por un periodo de cuatro meses, tras el cual todos los individuos se encontraban en proceso de descomposición, y durante ese periodo también presentó la tasa de crecimiento más baja, lo cual indica que en relación con las otras 5 parcelas, esta presentaba las condiciones menos óptimas para el establecimiento de las plántulas. La tasa de crecimiento de la parcela 5 presentó una tasa de deposición de sedimentos mayor que la estimada con la estaca control, lo cual resultó en valores negativos y absurdos sobre la tasa de crecimiento promedio. La topografía circundante a esta parcela pudo ocasionar una diferencia importante en la forma en que los sedimentos se depositaban en el área de estudio, ya que esta parcela se encontraba en una depresión, de suelo arenoso, que fácilmente puede ser removido bajo condiciones de inundación. Por ello, no es apropiado estimar la tasa de crecimiento, ya que es claro que hay una situación distinta en esta parcela debido a su topografía. En siguientes estudios se recomienda medir la tasa de sedimentación en cada parcela, con el fin de contar con un dato 
específico para cada una de las condiciones topográficas presentes.

Considerando que tras un año de seguimiento todas las parcelas se encontraron desprovistas de plántulas, es claro que el reclutamiento es un factor pivotal que limita la capacidad de regeneración natural en este ecosistema. Otros estudios han encontrado que la cobertura del dosel es un factor que limita el establecimiento de plántulas en los ecosistemas de manglar (Ellison \& Farnsworth, 1993; Farnsworth \& Ellison, 1996) y especialmente la dominancia de $A$. aureum es un factor que obstaculiza el proceso de regeneración natural es los ecosistemas de manglar (Srivastava et al., 1987; Mainardi-Grellet, 1995). Jiménez-Ramón (1988) reportan una densidad de plántulas establecidas exitosamente de 7 individuos $/ \mathrm{m}^{2}$ transcurridos 24 meses de monitoreo, lo cual es radicalmente distinto a los hallazgos de este estudio. En contraste, Jiménez, Lugo y Cintrón (1985) consideran que una tasa de supervivencia de propágalos de un $0.4 \%$ tras cinco años de monitoreo, es normal dentro de los ecosistemas de manglar. Jiménez y Soto (1985) describen que tanto $R$. racemosa como $P$. rhizophorae son especies que forman parte de la vegetación nuclear, la cual raramente se encuentra fuera de las zonas bajo influencia de la marea. Esta condición no es cierta para la ecosistema estudiado, lo cual confirma que en el momento en que este ecosistema se desarrolló, las condiciones eran distintas a las actuales, y posiblemente el área presentaba influencia directa por parte de la marea. Dado que las condiciones han cambiado, la capacidad de regeneración de este ecosistema se ha reducido.

Es necesario ahondar en la estructura demográfica de las poblaciones vegetales, con el fin de establecer claramente el nivel de regeneración natural presente en este ecosistema. Como nota preliminar en este sentido, se hace notar la aparente dominancia de individuos maduros y virtual ausencia de individuos juveniles en las zonas H2 Y B2 lo cual es coincidente con los resultados encontrados en las parcelas estudiadas, donde las tasas de supervivencia son prácticamente nulas, y posiblemente la tasa de reclutamiento es extremadamente baja. Se recomienda que futuros estudios incluyan parámetros relativos a la densidad, altura y DAP. Otro factor que no se midió en este estudio pero es importante mencionar, es la aparente muerte masiva de los árboles maduros de este ecosistema, fenómeno que en otros ecosistemas ha sido parcialmente atribuido a la acumulación de sedimentos (Jiménez et al., 1985; Elster, 2000), lo cual de ser confirmado, sería coincidente con los hallazgos de este estudio. Varios autores coinciden en que esta región sufre de altos niveles de sedimentación que afectan la salud del ecosistema. (UmañaVillalobos \& Springer, 2006; Flores-Guillen, 2001, citado en Sierra et al., 2007; Rojas \& Rodríguez, 2008), por lo tanto se concluye que ello constituye la principal causa de mortalidad de plántulas de mangle y baja capacidad de regeneración.

A pesar de las limitaciones de este estudio, es claro que las condiciones físicas actuales no favorecen la regeneración natural de los mangles, y en cambio se percibe una transición hacia un bosque continental, lo cual se mantendría si las condiciones actuales prevalecen. Evidencia de ello es la alta cobertura del helecho A. aureum, que se encuentra de forma natural en hábitat de transición entre el manglar y el bosque continental, debido a su condición halófito facultativo (Gokhale, Shaikh \& Chavan, 2011), así como baja la salinidad del manto freático y la poca o nula influencia directa de la marea. Otras variables que no fueron cuantificadas que podrían estar contribuyendo a la baja capacidad de regeneración natural, son la depredación (Clarke \& Myerscough, 1993) y la capacidad de dispersión de propágulos (Jiménez \& Sauter, 1991). Esta última puede ser de importancia para el ecosistema estudiado, ya que al no tener influencia de la marea, la capacidad de dispersión de los propágalos se ve severamente reducida al área directamente por debajo de los individuos reproductivos, donde la competencia intraespecífica es muy fuerte. Se espera que el área de estudio esté influenciado por variables similares que pueden ser estudiadas en futuras investigaciones. 
En conversaciones informales con personas locales y de la tercera edad (Anónimo, com. pers.), se menciona que aproximadamente hace 25 años, la pleamar se encontraba significativamente más cerca de los mangles adultos del estero Tortuga por lo que se considera importante que futuros estudios registren este parámetro, e incluso una comparación cronológica de imágenes satelitales podría sugerir que las condiciones que permitieron el desarrollo de los manglares del Estero Tortuga fueron distintas a las actuales. Debido a la naturaleza altamente dinámica de la zona de estudio, la influencia del río Térraba y las condiciones climáticas globales actuales, es posible que los fenómenos naturales extremos y el aumento de los niveles del mar, favorezcan una mayor influencia de la marea sobre el bosque de mangle estudiado, reestableciendo una de las condiciones necesarias para la regeneración del manglar. Esta condición se observa en las orillas del estero Tortuga, ubicadas en la zona NP3 (Fig. 1), donde tras el evento de inundación del 2010, se abrieron nuevos bancos de arena que ahora presentan una influencia directa de la marea y se observaron sectores de L. racemosa en estadíos juveniles. Ellison y Farnsworth (1993) y Krauss et al. (2008) reportan que para otras especies de mangle, la ausencia de dosel, favorece el crecimiento de los propágalos. Se recomienda realizar estudios de reclutamiento en estos sectores, especialmente porque es predecible que el alto aporte de sedimentos prevalezca, por lo tanto es importante registrar si esta especie es capaz de sobrellevar esta presión.

\section{Recomendaciones a las autoridades} competentes: Los resultados de esta investigación muestran que el Estero Tortuga debe ser tratado tanto a nivel local como regional. En primer lugar se debe procurar la actualización de sus límites para contar con un registro oficial del área que abarca este humedal y así facilitar un adecuado manejo de este ecosistema. La inclusión de las zonas de manglar que se encuentran catalogadas como no patrimonio son de alta prioridad ya que incluyen bosques de manglar desarrollados o en proceso regeneración natural. Se recomienda a las autoridades competentes, considerar la inclusión dentro del Patrimonio Natural del Estado, las zonas que presentan suelos hídricos y condición hídrica, pero que han sido recientemente deforestadas, ya que la vegetación hidrófila, sea de tipo manglar, bosque abnegado u otro, pueden volver a desarrollarse, si las perturbaciones humanas son controladas.

Este estudio colabora con mayor evidencia para justificar que el manejo de la cuenca Térraba Sierpe es prioritario, con el fin de disminuir la cantidad de sedimentos que esta deposita en los manglares de la zona. La capacidad de regeneración del bosque de mangle es limitada en algunos sectores del Estero Tortuga, lo cual se atribuye a las altas tasas de deposición de sedimento, pero se observa que existen otros sectores con alta capacidad de regeneración que podrían llegar a constituirse como los futuros manglares del Estero Tortuga, si se les presta la adecuada atención y protección.

\section{AGRADECIMIENTOS}

A Leo Whiteside por el apoyo financiero, Cristina Volkart y Alana Alonso por su asistencia en el campo, Aitor Aguirregoikoa por su apoyo técnico, y Frank Tortoriello, Alexia Maizel y Oscar Brenes por la oportunidad brindada.

\section{RESUMEN}

Este estudio se realizó entre Febrero 2011 y Enero 2012, en el bosque de mangle del Estero Tortuga. Se establecieron un total de seis parcelas, de $9 \mathrm{~m}^{2}$ cada una, con el fin de evaluar el porcentaje de supervivencia y la tasa de crecimiento de las plántulas de Rhizophora racemosa (G. Mey.) y Pelliciera rhizophorae (Triana \& Planch.). Al final del estudio todas las parcelas presentaron un $100 \%$ de mortalidad, lo que indica que la zona no está reclutando árboles jóvenes y por lo tanto no se está regenerando naturalmente. Los resultados de este estudio sugieren que la baja influencia de la marea, la baja salinidad del manto freático y la alta tasa de deposición de sedimentos, son las causas de la nula supervivencia de las plántulas y del deterioro de los individuos adultos. Se realizó un análisis comparativo de las zonas de manglar declaradas Patrimonio Natural de Estado y el ecosistema de manglar del Estero Tortuga, el cual muestra que existen áreas que no se encuentran 
protegidas bajo esta categoría. Se recomienda actualizar esta información con el fin de contar con un registro preciso sobre la ubicación de los manglares de la zona. Además, es prioritario que se aborde el manejo de la cuenca Térraba Sierpe, con el fin de disminuir la cantidad de sedimentos que se depositan en los manglares de la zona.

Palabras clave: Manglar, regeneración, supervivencia, plántula, Estero Tortuga, Rhizophora racemosa (G. Mey.), Pelliciera rhizophorae (Triana \& Planch.).

\section{REFERENCIAS}

ACOSA, The Nature Conservacy, UCI, \& ELAP. (2008). Documento para oficialización del plan de manejo Humedal Nacional Térraba Sierpe. Costa Rica: Sistema Nacional de Áreas de Conservación.

ACOSA. (2009). Acuerdo PNE 012-09: Certificación del Patrimonio Natural del Estado entre las desembocaduras del Río Tortuga y el Río Punta Mala. Costa Rica: Sistema Nacional de Áreas de Conservación.

Bosire, J. O., Kaino, J. J., Olagoke, A. O., Mwihaki, L. M., Ogendi, G. M., Kairo, J. G., ... \& Macharia, D. (2013). Mangroves in peril: unprecedented degradation rates of peri-urban mangroves in Kenya. Biogeosciences Discuss, 10, 16371-16404.

Bravo, J. \& Windevoxhel, N. J. (1997). Manual para la identificación y clasificación de humedales en Costa Rica. UICN - MINAE- Embajada Real de los Países Bajos. San José, Costa Rica. 37 p.

Clarke, P. J., \& Myerscough, P. J. (1993). The intertidal distribution of the grey mangrove (Avicennia marina) in southeastern Australia: The effects of physical conditions, interspecific competition, and predation on propagule establishment and survival. Australian Journal of Ecology, 18, 307-315.

Córdoba-Muñoz, R., Romero-Araya, J. C., \& Windevoxhel-Lora, N. J. (1998). Inventario de los humedales de Costa Rica. Embajada Real de los Países Bajos, Costa Rica: UICN, MINAE, SINAC.

Duke, N. C. (1992). Mangrove floristics and biogeography. In A. L. Robertson \& D. M. Alongi (Eds.), Coastal and Estuarine Studies: Tropical Mangrove Ecosystems (pp. 63-100). Washington DC: American Geophysical Union.

Ellison, A. M., \& Farnsworth, J. (1993). Seedling survivorship, growth and response to disturbance in Belizian mangal. American Journal of Botany, 80(10), 1137-1145.

Ellison, A. M. (2004). Wetlands of Central America. Wetlands Ecology and Management, 12(1), 3-55.

Elster, C. (2000). Reasons for reforestation success and failure with three mangrove species in Colombia. Forest Ecology and Management, 131, 201-214.
Farnsworth, E. J., \& Ellison, A. M. (1996). Sun-shade adaptability of the red mangrove, Rhizophora mangle (Rhizophoraceae): Changes through ontogeny at several levels of biological organization. American Journal of Botany, 83, 1131-1143.

Frazier, S. (1999). Visión General de los Sitios Ramsar: una sinopsis de los humedales de importancia internacional en el mundo. The Words, Berkshire, England: Wetlands International.

Gokhale, M. V., Shaikh, S. S., \& Chavan, N. S. (2011). Floral survey of wet coastal and associated ecosystems of Maharashtra. Indian Journal of Geo Marine Sciences, 40(5), 725-730.

Instituto Meteorológico Nacional (IMN). (2012). Estaciones Automáticas (Río Claro). Instituto Meteorológico Nacional, Costa Rica. Recuperado de http://www. imn.ac.cr/IMN/MainAdmin.aspx?_EVENTTARGE $\mathrm{T}=\mathrm{ClimaCiudad} \& \mathrm{CIUDAD}=13$

Jiménez, J. A., Lugo, A. E., \& Cintrón, G. (1985). Tree Mortality in Mangrove Forests. Biotropica, 17(3), 177-185.

Jiménez, J. A., \& Soto, R. (1985). Patrones regionales en la estructura y composición florística de los manglares de la Costa Pacífica de Costa Rica. Revista Biología Tropical, 33(1), 25-37.

Jiménez-Ramón, J. A. (1988). The dynamics of Rhizophora racemosa Meyer, forests on the Pacific coast of Costa Rica. Brenesia, 30, 1-12.

Jiménez, J. A., \& Sauter, K. (1991). Structure and dynamics of mangrove forests along a flooding gradient. Estuaries, 14, 49-56.

Krauss, K. W., Lovelock, C. E., McKee, K. L., LópezHoffman, L., Ewe, S. M. L., \& Sousa, W. P. (2008). Environmental drivers in mangrove establishment and early development: A review. Aquatic Botany, 89, 105-127.

Lahmann-Zeledón, E. J. (1999). La Reserva Forestal de Térraba-Sierpe, Costa Rica: un ejemplo de uso adecuado del manglar. In A. Yañez-Arancibia \& A. L. Lara-Domínguez (Eds.), Ecosistemas de Manglar en América Tropical (pp. 292-298). México, Costa Rica, Missouri, USA: Instituto de Ecología A. C., UICN/ ORMA, NOAA/NMFS.

Mainardi-Grellet, V. (1995). Estructura y composición florística de rodales con Pelliciera rhizophorae del manglar del Estero Guarumal, Sierpe, Costa Rica (Tesis de Maestría). Centro Agronómico Tropical de Investigación y Enseñanza, Costa Rica.

McKee, K. L., Cahoon, D. R., \& Feller, I. C. (2007). Caribbean mangroves adjust to rising sea level through biotic controls on change in soil elevation. Global Ecology and Biogeography, 16, 545-556. 
McLeod, E., \& Salm, R. V. (2006). Managing mangroves for resilience to climate change. Gland, Switzerland: IUCN.

Ministerio de Ambiente, Energía y Telecomunicaciones (MINAET). (2010). Decreto No. 35803. La Gaceta, 73. Costa Rica.

Pizarro, F., \& Angulo, H. (1994). Diagnóstico de los manglares de la costa Pacífica de Costa Rica. In D. O. Summan (Ed.), El Ecosistema de manglar en América Latina y la cuenca del Caribe: su manejo y conservación (pp. 34-63). Florida, USA: University of Miami and The Tinker Foundation.

Pizarro, F., Piedra, L., Bravo, J., Asch, J., \& Asch, C. (2004). Manual de procedimientos para el manejo de manglares en Costa Rica. Heredia, Costa Rica: EFUNA.

Rabinowitz, D. (1978). Dispersal properties of mangrove propagules. Biotropica, 10(1), 47-57.

Rojas, J. R., \& Rodríguez, O. (2008). Diversidad y abundancia ictiofaunística del río Grande de Térraba, sur de Costa Rica. Revista Biología Tropical, 56(3), 1429-1447.

Said-Mohamed, M. O., Neukermans, G., Gitundu-Kairo, J., Dahdouh-Guebas, F., \& Koedam, N. (2009). Mangrove forest in peri-urban setting the case of Mombasa Kenya. Wetlands Ecology and Management, 17, 243-255.

Sierra, C., Castillo, E., \& Arguedas, S. (2007). Documento de trabajo para el plan de manejo del Humedal Nacional Térraba-Sierpe: Diagnósticos biofísico, social, económico, productivo y análisis institucional. Serie Documental PMACOSA. (No. 42). Costa Rica.

Spalding, M., Kainuma, M., \& Collins, L. (2010). World atlas of mangroves. Malta: Earthscan, Gutenberg Press.

Srivastava, P. B. L., Keong, G. B., \& Muktar, A. (1987). Role of Achrostichum species in natural regeneration of Rhizophora species in Malaysia. Tropical Ecology, 28, 274-288.

Unión Internacional para la Conservación de la Naturaleza (UICN). (2002). Seguimiento de las directrices de la Convención RAMSAR en la planificación de los humedales de importancia internacional en Centroamérica. San José, Costa Rica: Unión Mundial para la Naturaleza, Oficina regional para Mesoamérica, Gobierno de Noruega.

Umaña-Villalobos, G., \& Springer, M. (2006). Variación ambiental en el río Grande de Térraba y algunos de sus afluentes, Pacífico sur de Costa Rica. Revista Biología Tropical, 54(1), 265-272.

Upadhyay, V. P., Ranjan, R., \& Singh, J. S. (2002). Humanmangrove conflicts: The way out. Current Science, 83(11), 1328-1336.

Uribe, M. (2010). Térraba Sierpe Wetland's Management plan: struggling for policy change and its implementation (Master's thesis). Vrije Universiteit, Amsterdam, Holland.

U. S. Geological Survey (USGS). (2014). Saline Water U.S Geological Survey. Retrieved from http://water.usgs. gov/edu/saline.html

Valiela, I., Bowen, J. L., \& York, J. K. (2001). Mangrove forests: one of the world's threatened major tropical environments. BioScience, 51(10), 807-815.

Villela, L. (1999). Memorias del Taller Conservación y Manejo de Humedales y Zonas Costeras en América Central: Metodologías y Prioridades. UICN, Guatemala.

Yáñez-Arancibia, A. \& Lara-Domínguez, A. L. (1999). Los manglares de América Latina en la encrucijada. In A. Yañez-Arancibia \& A. L. Lara-Domínguez (Eds.), Ecosistemas de Manglar en América Tropical (pp. 8-16). México, Costa Rica, Missouri, USA: Instituto de Ecología A. C., UICN/ORMA, NOAA/NMFS. 